\title{
Clinical significance of KAI1/CD82 protein expression in nasopharyngeal carcinoma
}

\author{
GENGMING WANG ${ }^{1,2}$, HAO JIANG $^{2}$, HONGBO XU $^{2}$, QIAN SUN ${ }^{2}$, YAN ZHOU $^{2}$, PING XIANG $^{3}$, \\ ZENONG CHENG ${ }^{4}$, YAJUN ZHANG ${ }^{2}$, YUFU ZHOU ${ }^{2}$, QING GUO $^{5}$, XINGLONG DU $^{6}$, \\ SHUXIU XU ${ }^{7}$, SHIYIN MA ${ }^{7}$ and ZHENDONG CHEN ${ }^{1}$ \\ ${ }^{1}$ Department of Oncology, The Second Affiliated Hospital of Anhui Medical University, Hefei, Anhui 230601; \\ ${ }^{2}$ Department of Radiotherapy, ${ }^{3}$ Central Laboratory and ${ }^{4}$ Department of Pathology, \\ The First Affiliated Hospital of Bengbu Medical College, Bengbu, Anhui 233004; ${ }^{5}$ Department of Radiotherapy, \\ Taizhou People's Hospital, Taizhou, Zhejiang 225300; ${ }^{6}$ Department of Radiotherapy, \\ The Second People's Hospital of Chuzhou, Chuzhou, Anhui 239000; ${ }^{7}$ Department of Otolaryngology, \\ The First Affiliated Hospital of Bengbu Medical College, Bengbu, Anhui 233004, P.R. China
}

Received February 4, 2014; Accepted October 15, 2014

DOI: $10.3892 / \mathrm{ol} .2015 .2891$

\begin{abstract}
The aim of this study was to investigate KAI1/CD82 protein expression in human nasopharyngeal carcinoma (NPC) cell lines and human NPC tissues. Immunohistochemistry and western blot analysis were used to detect the localization and expression levels of the KAI1/CD82 protein in five human NPC cell lines. Immunohistochemistry was also conducted to detect the expression of the KAI1/CD82 protein in 70 NPC tissues and 30 non-neoplastic nasopharyngeal tissues. The levels of $\mathrm{KAI} 1 / \mathrm{CD} 82$ protein expression were found to decrease as the metastatic potential of cells increased. The expression rate of KAI1/CD82 protein in the NPC tissues (44.3\%) was significantly lower than that in the non-neoplastic nasopharyngeal tissues $(70.0 \%)(\mathrm{P}<0.05)$. KAI1/CD82 protein expression in the NPC tissues was not associated with clinical parameters, including gender, age, histological type and $\mathrm{T}$ stage, and the positive expression of KAI1/CD82 decreased with increased $\mathrm{N}$ staging. The level of KAI1/CD82 protein expression was increased in different human NPC cell lines. The KAI1/CD82 gene was highly expressed in cells with low metastatic potential, while low expression was observed in cells with a high metastatic potential. In addition, the KAI1/CD82 gene was expressed at low levels in nasopharyngeal carcinoma tissues, while high expression was identified in non-neoplastic nasopharyngeal tissues, and was associated with lymph node metastasis. These results indicated that the KAI1/CD82 gene
\end{abstract}

Correspondence to: Dr Zhendong Chen, Department of Oncology, The Second Affiliated Hospital of Anhui Medical University, 678 Lotus Road, Hefei, Anhui 230601, P.R. China

E-mail: zhendongchencn@163.com

Key words: expression, nasopharyngeal carcinoma, KAI1/CD82, protein, metastasis may be involved in the occurrence, development and metastasis of nasopharyngeal carcinoma.

\section{Introduction}

Nasopharyngeal carcinoma (NPC) is a highly invasive malignant tumor, which occurs in the nasopharynx. Although it is rare in the majority of countries (1), the incidence and mortality rates of NPC are markedly high among Southern Chinese populations. The five-year survival rate following combined treatment with radiotherapy and adjuvant cisplatin chemotherapy is $50-60 \%$ and the rates of five-year cumulative local relapse and distant metastasis are 20-30 and 20-25\%, respectively (2). Therefore, investigating distant metastasis in NPC, and minimizing the occurrence of distant metastasis have become key to further improve the efficacy of NPC treatment and, thus, have important practical significance. The KAI1/CD82 gene, which belongs to the transmembrane 4 superfamily (TM4SF), was identified by Dong et al (3) in 1995. The inhibitory effects of the TM4SF protein on tumor metastasis have been demonstrated (4); its cell-membrane location and extensive glycosylation leads to cell-cell and cell-extracellular matrix interactions, which subsequently affect tumor metastasis. These interactions are extremely important with regard to the invasion and metastasis of tumors. In this study, immunohistochemistry and western blot analysis were used to detect the levels of KAI1/CD82 protein expression in five different human NPC cell lines, CNE-1, CNE-2Z, SUNE-1, SUNE-1-5-8F and SUNE -1-6-10B, and immunohistochemistry was also performed to detect the KAI1/CD82 protein expression in NPC and non-neoplastic nasopharyngeal tissues. The association between abnormal KAI1/CD82 gene expression in NPC and patient age, gender, histological type, $\mathrm{T}$ staging and lymph node staging were analyzed. The aim of this study was to investigate novel methods, which may improve the treatment efficacy and the prognosis, as well as reduce the occurrence of metastasis. 


\section{Materials and methods}

Specimens. The human NPC cell lines, CNE-1, CNE-2Z, SUNE-1, SUNE-1-5-8F and SUNE -1-6-10B, with various metastatic characteristics were purchased from Hefei Shengmai Reagent Co., Ltd (Hefei, China). The detailed metastatic characteristics and levels of differentiation are shown in Table I.

A total of 70 archived paraffin-embedded NPC specimens were obtained from the Department of Pathology. The First Affiliated Hospital of Bengbu Medical College (Bengbu, China) between February 2007 and August 2010. The clinical data of all patients were complete and no patients had received radiotherapy or chemotherapy prior to biopsy. In addition, 30 archived paraffin-embedded non-neoplastic nasopharyngeal tissue specimens served as the control group, which were all samples of nasopharyngeal chronic mucosal inflammation, with or without the lymphoid hyperplasia. This study was conducted in accordance with the Declaration of Helsinki and with approval from the ethics committee of The First Affiliated Hospital of Bengbu Medical College. Written informed consent was obtained from all participants.

Immunohistochemical detection of KAII/CD82 expression in human NPC cell lines. After anabiosis, medium-changing and three passages, the five NPC cell lines were seeded in the pre-sterile-coverslip-paved six-well plates at a density of $\sim 5 \times 10^{4}$ cells $/ \mathrm{ml}$. Following incubation at $37^{\circ} \mathrm{C}$ in an atmosphere of $5 \% \mathrm{CO}_{2}$ for $48 \mathrm{~h}$, the coverslips were removed and immunohistochemical staining with Histostain ${ }^{\mathrm{TM}}$-Plus kits (Beijing Zhongshan Biotechnology Co., Ltd., Beijing, China) was performed at room temperature. The appearance of brown granules on the cell surface and inside the cytoplasm was considered to indicate positive KAI1/CD82 expression. A total of four cell-attached coverslips of the KAI1/CD82 protein from each cell line were randomly selected, and 500 cells in each cell-attached coverslip were randomly selected under a microscope (BX50; Olympus, Tokyo, Japan; magnification, $\mathrm{x} 400$ ); the total number of cells in the four cell-attached coverslips was 2,000. The number of positive cells among the 500 randomly selected cells in each of the above four cell-attached coverslips was counted to calculate the positive KAI1/CD82 expression rate, with the following formula: Positive rate of KAI1/CD82 expression $(\%)=$ number of positive cells / total number of cells counted. According to the number of cells and the percentage of positive cells, the results were classified into four grades: No expression (-), no positive cells in the cell-attached coverslip; low expression (+), $>0 \%$ and $<24 \%$ positive cells; moderate expression $(++), 25-50 \%$ positive cells; and high expression (+++), $>50 \%$ positive cells.

The $\chi^{2}$ test was used to compare the positive rates of KAI1/CD82 protein among the cell line with the lowest metastatic potential (SUNE-1-6-10B) and the other cell lines with higher metastatic potentials.

Western blot analysis. A total of $1 \times 10^{7}$ cells of each of the five NPC cell lines, which were wall-adherent, were collected. Next, $200 \mu 1$ cell lysate was added for the 30 min lysis on ice, followed by centrifugation at $2,862 \times \mathrm{g}$ for $30 \mathrm{~min}$ at $4^{\circ} \mathrm{C}$ (5810R, Eppendorf, Hamburg, Germany). The supernatant was obtained, and the protein concentration was determined using the Coomassie Brilliant Blue assay (G-250, Beijing
Table I. Differentiation status and metastatic characteristics of the five cell lines used in this study.

\begin{tabular}{lll}
\hline Cell line & \multicolumn{1}{c}{$\begin{array}{c}\text { Level of } \\
\text { differentiation }\end{array}$} & $\begin{array}{c}\text { Metastatic } \\
\text { characteristics }\end{array}$ \\
\hline SUNE-1 & Poorly differentiated & SqCa high \\
CEN-2Z & Poorly differentiated & SqCa high \\
SUNE-1-5-8F & Poorly differentiated & SqCa extremely high \\
SUNE-1-6-10B & Poorly differentiated & SqCa low \\
CEN-1 & Highly differentiated & SqCa middle \\
\hline
\end{tabular}

Extremely high, high, middle and low refer to the degree of metastasis. $\mathrm{SqCa}$, squamous cell carcinoma.

Zhongshan Biotechnology Co.,Ltd.). The protein concentration was then adjusted to $50 \mu \mathrm{g} / \mu \mathrm{l}$. Next, $10 \%$ SDS-PAGE electrophoresis was performed for $3 \mathrm{~h}$ to separate the proteins, which were then transferred to nitrocellulose membranes. After three washes with phosphate buffered-saline (PBS; Fuzhou Maixin Biotechnology Development Co., Ltd., Fuzhou, China) for $15 \mathrm{~min}$ each, $1 \%$ bovine serum albumin (Fuzhou Maixin Biotechnology Development Co., Ltd.) was used to block non-specific antigen activity for $2 \mathrm{~h}$. After blocking, the membranes were incubated with mouse anti-human KAI1/CD82 monoclonal primary antibody (1:250; sc-17752; Santa Cruz Biotechnology, Inc., CA, USA) overnight at $4^{\circ} \mathrm{C}$, followed by washing with PBS. The alkaline goat anti-rat polyclonal phosphatase-labeled secondary antibody (1:200; PV6002, Beijing Zhongshan Biotechnology Co., Ltd.; generaltype kit; Fuzhou Maixin Biotechnology Development Co., Ltd.) was added, followed by incubation at room temperature for $2 \mathrm{~h}$. NBT/BCIP coloration was conducted for $15 \mathrm{~min}$ (kits were provided by Fuzhou Maixin Biotechnology Development Co., Ltd., Fuzhou, China). When clear brown bands, which indicate positive staining, were observed on the membranes, the coloration was terminated, followed by rinsing, drying and preservation of the membranes.

Immunohistochemical detection of KAII/CD82 protein expression. All NPC and non-neoplastic nasopharyngeal tissue specimens were fixed in $10 \%$ formalin, embedded in paraffin and cut into $4 \mu \mathrm{m}$-thick serial sections. After dewaxing and hydration, antigen retrieval in potassium citrate buffer was performed on the sections using a microwave. Horseradish peroxidase (Fuzhou Maxim Bioengineering Co. Ltd., Fuzhou, China) was then added to label the avidin, followed by staining with 3,3'-Dimethylbenzidine (Fuzhou Maixin Biotechnology Development Co., Ltd.). After rinsing with water, the specimens were re-stained with hematoxylin (Fuzhou Maixin Biotechnology Development Co., Ltd.), followed by an additional rinse with water. The specimens were then dehydrated in ethanol and cleared in xylene (Wuxi Jingke Chemical Co., Ltd., Wuxi, China), followed by mounting with neutral gum (Fuzhou Maixin Biotechnology Development Co., Ltd.). PBS was used to replace the primary antibody for the blank control group. The nasopharyngeal tissues were observed under a microscope (BX50; Olympus), 
Table II. Comparison of KAI1/CD82 protein positive expression rates in five cell lines.

\begin{tabular}{|c|c|c|c|c|c|}
\hline Cell line & Cells, $\mathrm{n}$ & Positive cells, $\mathrm{n}$ & Positive rate $(\%)^{\mathrm{a}}$ & $\chi^{2}$ & P-value \\
\hline SUNE-1-6-10B & 2000 & 1410 & 70.5 & - & - \\
\hline $\mathrm{CNE}-1^{\mathrm{a}}$ & 2000 & 814 & 40.7 & 459 & $4.89 \times 10^{-83}$ \\
\hline CNE-2Z & 2000 & 803 & 40.2 & 467 & $1.29 \times 10^{-101}$ \\
\hline SUNE-1 ${ }^{\mathrm{a}}$ & 2000 & 736 & 36.8 & 526 & $6.77 \times 10^{-100}$ \\
\hline SUNE-1-5-8F & 2000 & 394 & 19.7 & 1196 & $6.41 \times 10^{-248}$ \\
\hline
\end{tabular}

${ }^{\mathrm{a} C}$ Compared with SUNE-1-6-10B (tumorigenesis and low metastatic), $\mathrm{P}<0.05$.

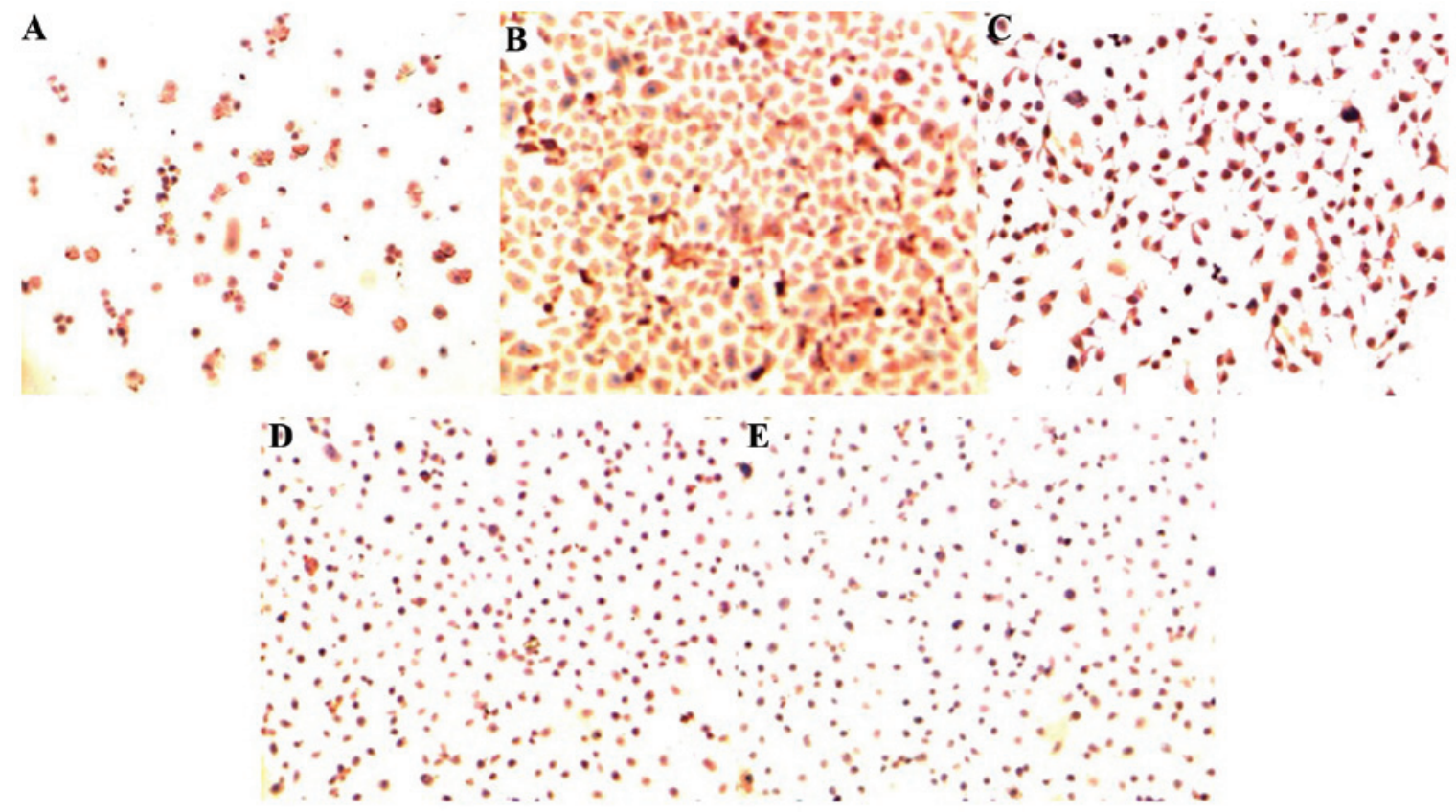

Figure 1. KAI1/CD82 protein expression in human nasopharyngeal carcinoma in (A) SUNE-1-6-10B, (B) CNE-1, (C) CNE-2Z, (D) SUNE-1 and (E) SUNE-1-5-8F cell lines with different metastatic potentials (magnification, $\mathrm{x} 100$ ).

and the appearance of brown particles in the cytoplasm was considered to indicate positive expression. A total of four different views at high magnification were performed to count 100 cells in each view, which were divided into $(+)$ to $(+++)$ according to the percentage of positive cells, which was scored as follows: no positive cells (-); 1-9\% positive cells (+); $10-50 \%$ positive cells $(++) ;>50 \%$ positive cells $(+++)$. The $(+)$ to $(+++)$ scores were classified as positive expression, while (-) score was considered as negative expression.

Statistical analysis. The western blotting data were analyzed using one-factor analysis of variance and the immunohistochemistry data were analyzed by the $\chi^{2}$ test. All data were analyzed using SPSS software, version 17.0 (SPSS, Inc., Chicago, IL, USA) and $\mathrm{P}<0.05$ was considered to indicate a statistically significant difference.

\section{Results}

Immunohistochemical detection of KAII/CD82 protein levels in the five NPC cell lines. The immunohistochemical results showed that the KAI1/CD82 protein was significantly expressed in the membrane and/or cytoplasm of all five NPC cell lines, with the positive signal of brown particles. The results revealed that the KAI1/CD82 protein was located in the membrane and/or cytoplasm in all five cell lines. KAI1/CD82 was highly expressed in the cytoplasm and membrane of the SUNE-1-6-10B cell line (tumorigenesis and low metastatic potential), while low expression was exhibited in the cytoplasm and membrane of the SUNE-1-5-8F cell line (high tumorigenesis and metastatic potential). In the remaining cell lines, the expression varied (Fig. 1). The positive rate of KAI1/CD82 protein expression in each cell line was calculated and the $\chi^{2}$ test was performed, which revealed that the positive expression rate of KAI1/CD82 protein in the SUNE-1-6-10B cells (tumorigenesis and low metastatic potential) was significantly higher than that in the cell lines with a higher metastatic potential $(\mathrm{P}<0.01)$.

SPSS 17.0 software was used to perform the completely randomized-design $\chi^{2}$ test, which revealed that the positive expression rate in the SUNE-1-6-10B cells was significantly different when compared with the other groups $(\mathrm{P}<0.05)$ 
Table III. Expression level changes of KAI1/CD82 gene in different human nasopharyngeal carcinoma cell lines.

\begin{tabular}{lll}
\hline Group & $\mathrm{n}$ & \multicolumn{1}{c}{ OD $^{\mathrm{a}}$} \\
\hline SUNE-1-6-10B & 3 & $1594 \pm 13.95$ \\
CNE-1 & 3 & $1453 \pm 11.34$ \\
CNE-2Z & 3 & $1326 \pm 11.78$ \\
SUNE-1 & 3 & $1314 \pm 9.09$ \\
SUNE-1-5-8F & 3 & $1245 \pm 10.42$ \\
\hline
\end{tabular}

${ }^{a}$ Mean \pm SD. n, number of experimental repeats; OD, optical density.

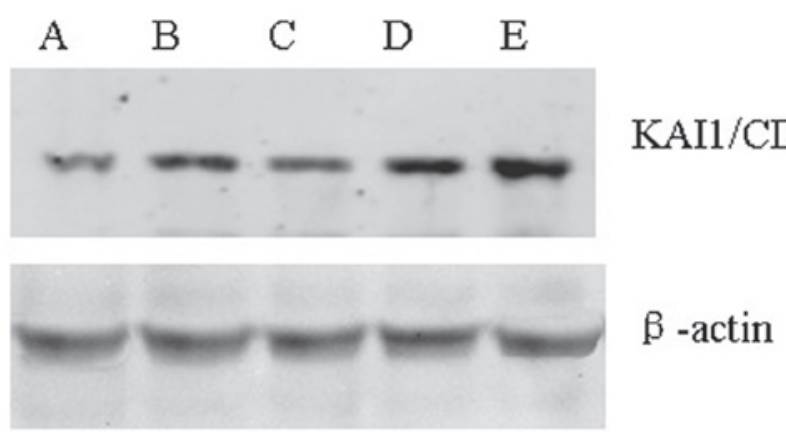

Figure 2. KAI1/CD82 gene expression in different human nasopharyngeal carcinoma cell lines. (A) SUNE-1-5-8F, (B) CNE-2Z, (C) SUNE-1, (D) CNE-1 and (E) SUNE-1-6-10B.
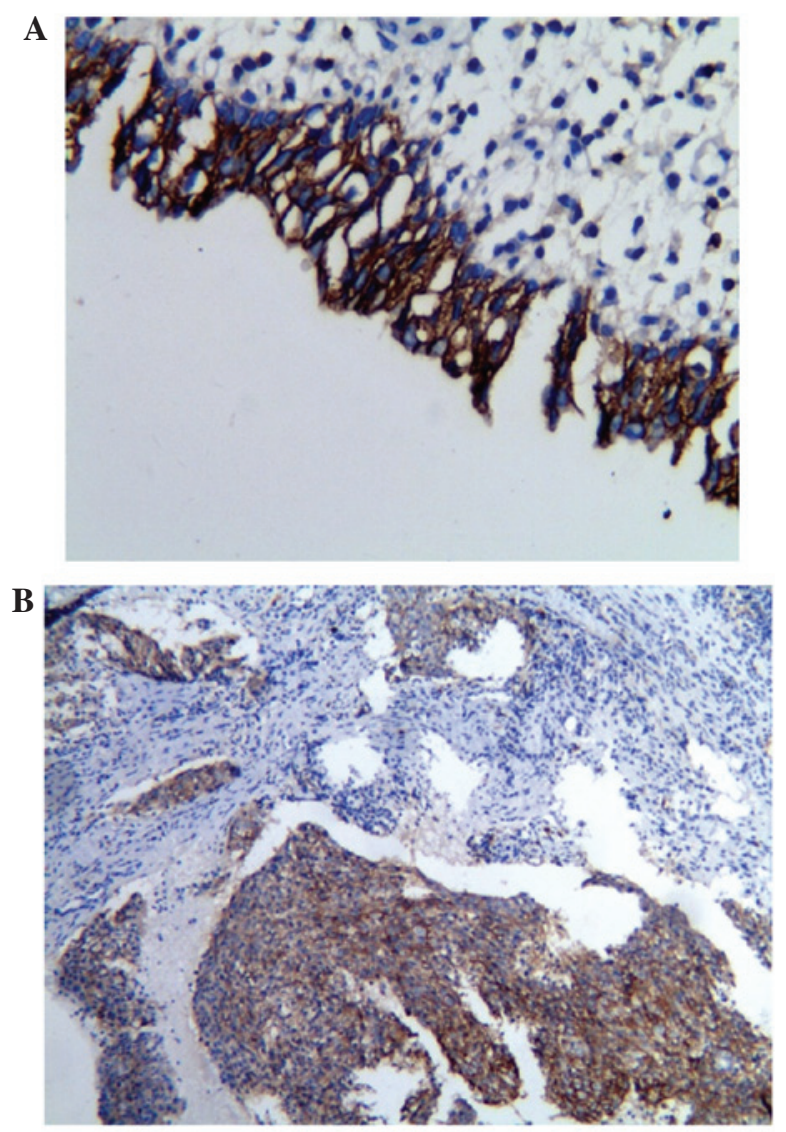

Figure 3. Positive KAI1/CD82 protein expression was identified in (A) nonneoplastic nasopharyngeal and (B) NPC tissues by immunohistochemistry. (magnification: A, x40; B, x10).
(Table II). Furthermore a significant difference in expression rate was identified between the CNE-1 and SUNE-1 cell lines, and between the CNE-2Z and SUNE-1-5-8F cell lines $(\mathrm{P}<0.05)$, while no significant difference was identified between the SUNE-1 and CNE-2Z cell lines $(\mathrm{P}>0.05)$.

Western blot analysis of KAII/CD82 protein levels in the five NPC cell lines. Western blot analysis revealed that the KAI1/CD82 protein expression levels differed in the five NPC cell lines (Fig. 2). $\beta$-actin was used as the internal reference. The results revealed that compared with the SUNE-1-5-8F cell line, the protein expression of KAI1/CD82 in the SUNE-1-6-10B and CNE-1 cell lines was significantly increased $(\mathrm{P}<0.05)$ (Table III).

SPSS 17.0 software was used to perform the LEVENE homogeneity of variance test, the results of which were $\mathrm{F}=0.485$ and $\mathrm{P}=0.747(\mathrm{P}>0.05)$, indicating that the data of the five groups exhibited homogeneity of variance. Furthermore, the completely randomized-design analysis of variance results were $\mathrm{P}=0.000(\mathrm{P}<0.05)$ and $\mathrm{F}=315.775$; therefore, it was considered in general that the data obtained from the five groups were different. The pairwise comparison with least significant difference and Student-Newman-Keuls methods identified no significant difference in the protein expression between the CNE-2Z and SUNE-1 cell lines $(\mathrm{P}=0.195$ and $\mathrm{P}>0.05$, respectively); however, the pairwise comparisons between the other groups identified a significant difference $(\mathrm{P}<0.05)$.

Expression of the KAII/CD82 protein in NPC and non-neoplastic nasopharyngeal tissues. The immunohistochemical detection of the KAI1/CD82 protein expression in the two groups revealed that the positive expression rate of the KAI1/CD82 protein in the non-neoplastic nasopharyngeal tissues $(21 / 30 ; 70.0 \%)$ was significantly higher than that of NPC group $(31 / 70 ; 44.3 \%)$, and the $\chi^{2}$ test revealed a statistically significant difference in KAI1/CD82 protein expression between the two groups (Fig. 3) $(\mathrm{P}<0.05)$.

Association between KAII/CD82 protein expression and clinical factors. The expression of KAI1/CD82 protein in NPC was not found to correlate with the clinical characteristics of patients, including age, histological type and T staging, which was determined according to the International Union Against Cancer's (UICC) TNM staging system (5). However, the expression of KAI1/CD82 protein expression was found to correlate with lymph node metastasis. The positive expression rate of KAI1/CD82 protein in patients without lymph node metastasis $\left(\mathrm{N}_{0}\right)$, according to the UICC TNM staging system (5), was $68.4 \%(13 / 19)$, which was higher than that $(35.3 \%, 18 / 51)$ in the patients with the cervical lymph node metastasis $\left(\mathrm{N}_{1-3}\right)$, and this difference was statistically significant $(\mathrm{P}<0.05)$. With increased $\mathrm{N}$ staging, the KAI1/CD82 protein expression decreased $\left(\mathrm{N}_{0}, 68.4 \% ; \mathrm{N}_{1}, 43.8 \% ; \mathrm{N}_{2}\right.$, $33.3 \%$; and $\mathrm{N}_{3}, 25.0 \%$ ) (Table IV).

\section{Discussion}

Metastasis refers to the process by which malignant cells detach from the primary tumor and migrate to a secondary 
Table IV. Association between KAI1/CD82 protein expression and patient clinical parameters.

\begin{tabular}{|c|c|c|c|c|c|}
\hline \multirow[b]{2}{*}{ Clinical parameter } & \multirow[b]{2}{*}{$\mathrm{n}(\%)$} & \multicolumn{2}{|c|}{$\begin{array}{c}\mathrm{KAI}-1 / \mathrm{CD} 82 \\
\text { protein expression }\end{array}$} & \multirow[b]{2}{*}{$\chi^{2}$} & \multirow[b]{2}{*}{ P-value } \\
\hline & & + & - & & \\
\hline Gender & & & & 0.320 & 0.572 \\
\hline Male & $32(45.7)$ & 13 & 19 & & \\
\hline Female & $38(54.3)$ & 18 & 20 & & \\
\hline Age, years & & & & 0.170 & 0.681 \\
\hline$\leq 50$ & $41(58.6)$ & 19 & 22 & & \\
\hline$>50$ & $29(41.4)$ & 12 & 17 & & \\
\hline Pathological type & & & & 0.854 & 1.000 \\
\hline Squamous cell carcinoma & $69(98.6)$ & 32 & 37 & & \\
\hline Adenocarcinoma & $1(1.4)$ & 0 & 1 & & \\
\hline T staging & & & & 0.797 & 0.372 \\
\hline $\mathrm{T} 1-\mathrm{T} 2$ & $21(30.0)$ & 11 & 10 & & \\
\hline $\mathrm{T} 3-\mathrm{T} 4$ & $49(70.0)$ & 20 & 29 & & \\
\hline $\mathrm{N}$ staging & & & & 6.157 & 0.013 \\
\hline $\mathrm{N}_{0}$ & $19(27.1)$ & 13 & 6 & & \\
\hline $\mathrm{N}_{1}$ & $16(22.9)$ & 7 & 9 & & \\
\hline $\mathrm{N}_{2}$ & $27(38.6)$ & 9 & 18 & & \\
\hline $\mathrm{N}_{3}$ & $8(11.4)$ & 2 & 6 & & \\
\hline
\end{tabular}

tissue or organ via a variety of mechanisms. The cells continue to proliferate and grow, finally forming a secondary tumor that exhibits the same features as the primary tumor. Metastasis is one of the basic biological characteristics of malignant tumors, and the reason for the failure of treatment in the majority of cancer patients. Therefore, controlling metastasis is the key to improving the prognosis of cancer patients (6).

The KAI1/CD82 gene is located on the human chromosome 11p11.2 (3), which consists of 10 exons and nine introns $(\sim 80 \mathrm{~kb})(3)$. The structure of the product of the KAI1 gene is the same as CD82 and, thus, KAI1 is a member of the TM4SF family. The KAI1/CD82 gene exhibits an inhibitory function with regard to tumor metastasis, and this inhibition has been confirmed in a number of studies investigating malignant cancer (7-10). However, this inhibitory mechanism remains unclear, and previous studies have shown that multiple factors are involved, which may exert effects on their own or in combination, including the integrin family (11), epidermal growth factor receptor (12), EW12/PGRL (13), KITENIN $(14,15)$ and protein kinase C (16).

An initial study indicated that the KAI1/CD82 gene exhibited the inhibition specifically towards the metastasis of prostate cancer (17); however, later studies have revealed that the inactivation of this gene occurs in a number of other malignant tumors, including thyroid (14), breast (18-20), endometrial (16), laryngeal (21), colon (22), gastric $(7,23)$, gallbladder (24), liver (25), kidney (26), bladder (27) and prostate (28) cancer. In addition, these studies also demonstrated that the inactivation of the KAI1/CD82 gene was associated with tumor metastasis.
Although the KAI1/CD82 gene has been reported to be involved in numerous other cancer types, its involvement in NPC is largely unclear. In the present study, immunohistochemistry and western blot analysis were performed to investigate the expression levels of the KAI1/CD82 gene in five different NPC cell lines, which exhibited different metastatic characteristics. The KAI1/CD82 protein levels were found to correlate with the metastatic characteristics of the NPC cell lines. The positive expression rate of KAI1/CD82 in NPC was lower than that in the normal nasopharyngeal tissues, indicating that the KAI1/CD82 gene may be involved in the occurrence and development of NPC. In NPC, the underexpression of the KAI1/CD82 protein was found to correlate with lymph node metastasis. Furthermore, with the progression of $\mathrm{N}$ staging, the expression rate of KAI1/CD82 protein was found to gradually decline, indicating that the underexpression of KAI1/CD82 protein was associated with NPC metastasis, and that KAI1/CD82 may be involved in the metastasis of NPC.

Controlling tumor metastasis is a major focus of cancer research; tumor metastasis is the main reason for treatment failure and patient mortality, and there are currently difficulties with regard to the treatment of malignant cancer. Therefore, future studies investigating the tumor metastasis-related genes are required to improve the efficacy of tumor treatment. Since the follow-up period in the present study was short, the association between KAI1/CD82 gene expression and the treatment and prognosis of NPC was not analyzed. Further study is required to elucidate the true association between KAI1/CD82 expression and the biological behaviors of NPC. 


\section{References}

1. Lo KW, To KF and Huang DP: Focus on nasopharyngeal carcinoma. Cancer Cell 5: 423-428, 2004.

2. Siddique MA, Sabur MA, Kundu SC, et al: Difficulty in diagnosis of nasopharyngeal carcinoma. Mymensingh Med J 21: 158-161, 2012.

3. Dong JT, Lamb PW, Rinker-Schaefer CW, et al: KAI-1, a metastasis suppressor gene for prostate cancer on human chromosome 11p11.2. Science 268: 884-886, 1995.

4. Takeda T, Hattori N, Tokuhara T, et al: Adenoviral transduction of MRP-1/CD9 and KAI-1/CD82 inhibits lymph node metastasis in orthotropic lung cancer model. Cancer Res 67: 1744-1749, 2007.

5. Teo PM, Leung SF, Yu P, Tsao SY, Foo W and Shiu W: A comparison of Ho's, International Union Against Cancer, and American Joint Committee stage classifications for nasopharyngeal carcinoma. Cancer 67: 434-439, 1991.

6. Brierly JD, Catton PA, O'Sullivan B, et al: Accuracy of recorded tumor, node, and metastasis stage in a comprehensive cancer center. J Clin Oncol 20: 413-419, 2002.

7. Tsutsumi S, Shimura T, Morinaga N, et al: Loss of KAI-1 expression in gastric cancer. Hepatogastroenterology 52: 281-284, 2005

8. Yang X, Wei LL, Tang C, Slack R, Mueller S and Lippman ME: Overexpression of KAI1 suppresses in vitro invasiveness and in vivo metastasis in breast cancer cells. Cancer Res 61: 5284-5288, 2001.

9. Huang CI, Kohno N, Ogawa E, Adachi M, Taki T and Miyake M: Correlation of reduction in MRP-1/CD9 and KAIl/CD82 expression with recurrences in breast cancer patients. Am J Pathol 153: 973-983, 1998.

10. Takaoka A, Hinoda Y, Sato S, Itoh F, Adachi M, Hareyama M and Imai K: Reduced invasive and metastatic potentials of KAI-1 transfected melanoma cells. Jpn J Cancer Res 89: 397-404, 1998.

11. He B, Liu L, Cook GA, et al: Tetraspanin CD82 attenuates cellular morphogenesis through down-regulating integrin alpha6-mediated cell adhesion. J Biol Chem 280: 3346-3354, 2005.

12. Charrin S, Manie S, Oualid M, et al: Differential stability of tetraspanin/tetraspanin interactions: role of palmitoylation. FEBS Lett 516: 139-144, 2002.

13. Zhou B, Liu L, Reddivari M and Zhang XA: The palmitoylation of metastasis suppressor KAI-1/CD 82 is important for its motility-and invasiveness-inhibitory activity. Cancer Res 64 : 7455-7463, 2004.

14. Ito Y, Yoshida H, Uruno T, et al: KAI-1 expression in tlyroidneoplasmg: its linkage with clinic opathologic features in papillary carcinoma. Pathol Res Pract 199: 79-83, 2003.

15. Chen Z, Mustafa T, Trojanowicz B, et al: CD82 and CD63 in thyroid cancer. Int J Mol Med 14: 517-527, 2004.
16. Liu FS, Dong JT, Chen JT, et al: KAI1 metastasis suppressor protein is down-regulated during the progression of human endometrial cancer. Clin Cancer Res 9: 1393-1398, 2003.

17. Lijovic M, Somers G and Frauman AG: KAIl/CD82 Protein expression in primary prostate cancer and in BPH associated with cancer. Cancer Detect Prev 26: 69-77, 2002.

18. Christgen M, Bruchhardt H, Ballmaier M, et al: KAI-1/CD82 is a novel target of estrogen receptor-mediated gene repression and down regulated in primary human breast cancer. Int J Cancer 123: 2239-2246, 2008.

19. Christgen M, Christgen H, Heil C, et al: Expression of KAI1/CD82 in distant metastases from estrogen receptor-negative breast cancer, Inhibitory effect of KAI-1 gene on breast cancer cell growth in vitro. Cancer Sci 100: 1767-1771, 2009

20. Malik FA, Sanders AJ, Kayani MA and Jiang WG: Effect of expressional alteration of KAI-1 on breast cancer cell growth, adhesion, migration and invasion. Cancer Genomics Proteomics 6: 205-213, 2009.

21. Zhang BH, Liu W, Li L, et al: KAI1/CD82 and MRP1/CD9 serve as markers of infiltration, metastasis, and prognosis in laryngeal squamous cell carcinomas. Asian Pac J Cancer Prev 14: 3521-3526, 2013.

22. Ma ZB, Li K, Wang J and Guo GH: Role of KAI1/CD82 polymorphisms in colon cancer risk in Han Chinese population. Med Oncol 30: 668-672, 2013.

23. Knoener M, Krech T, Puls F, et al: Limited value of KAI1/CD82 protein expression as a prognostic marker in human gastric cancer. Dis Markers 32: 337-342, 2012.

24. Jiang WX, Song BG and Wang PJ: Expression of nm23, KAI1 and spiral computed tomography findings in primary gallbladder carcinoma. Chin Med J 122: 2666-2668, 2009.

25. Guo C, Liu QG, Zhang L, Song T and Yang X: Expression and clinical significance of p53, JunB and KAI1/CD82 in human hepatocellular carcinoma. Hepatobiliary Pancreat Dis Int 8: 389-396, 2009.

26. Yusenko MV and Kovacs G: Identifying CD82 (KAI1) as a marker for human chromophobe renal cell carcinoma. Histopathology 55: 687-695, 2009.

27. You J, Madigan MC, Rowe A, et al: An inverse relationship between KAI1 expression, invasive ability, and MMP-2 expression and activity in bladder cancer cell lines. Urol Oncol 9: 1324-1330, 2010.

28. Lee HA, Park I, Byun HJ, et al: Metastasis suppressor KAI1/CD82 attenuates the matrix adhesion of human prostate cancer cells by suppressing fibronectin expression and $\beta 1$ integrin activation. Cell Physiol Biochem 27: 575-586, 2011. 